\title{
Impact of Parity on Fracture Risk after Menopause: A Sys- tematic Review
}

\section{Demba Diédhiou ${ }^{1,2}$, Georges Weryha ${ }^{1}$, Anna Angelousi', Mikaël Agopiantz', Saïd Norou Diop², Olivier Morel'3 , Marie Laure Schweitzer ${ }^{4}$ and Cedric Baumann ${ }^{4}$}

${ }^{1}$ Department of Endocrinology, University Hospital Center of Nancy, Lorraine, Vandoeuvre-Les-Nancy, France

${ }^{2}$ Department of Internal Medicine II, University Hospital Center of Dakar, Cheikh Anta Diop University, Dakar, Senegal

${ }^{3}$ Department of Obstetrics and Medical Gynecology, University Hospital Center of Nancy, Lorraine University, Vandoeuvre-Les-Nancy, France

${ }^{4}$ Department of Epidemiology and Clinical Evaluation, University Hospital Center of Nancy, Lorraine University, Vandoeuvre-Les-Nancy, France

\begin{abstract}
\section{Background}

Pregnancy and breastfeeding cause temporal bone resorption and can play a role in clinical expression of osteoporosis disease. Objective was to estimate the association between parity and risk of osteoporotic fracture after menopause.

\section{Methods}

We performed a systematic review through a search of PubMed, Cochrane, Embase databases from January 1, 1980 through January 31,2016 . We included all studies that evaluated the link between parity and post-menopausal osteoporotic fracture using univariate and/or multivariate analysis.

\section{Results}

Among 29 studies, a positive effect of parity was found in $7 / 13$ prospective, $1 / 7$ transversal and $2 / 10$ retrospective studies. Three studies out of 5 found that parity had a protective role against the risk for any fracture $(H R=0.94[0.90-0.99], O R=0.41[0.28-0.61]$ and $\mathrm{OR}=0.90[0.84-0.99])$. For hip fracture, a protective effect was found in 5 studies out of 17 and concerned women with $\geq 2$ children in two studies $(\mathrm{OR}=0.75[0.62-0.91], \mathrm{RR}=0.5[0.32-0.79])$. For vertebral fracture, 1 study out of 8 reported a significantly reduced risk in women with $\geq 2$ children $(H R=0.44[0.26-0.76])$. Wrist fracture risk was
\end{abstract}

${ }^{*}$ Corresponding author: Georges Weryha, Department of Endocrinology, University Hospital Center of Nancy, CHU Brabois Adultes, Rue du Morvan, 54500 Vandoeuvre Les Nancy, France, Tel: +33 608335915; Fax: +33 383153440; Email: g.weryha@chu-nancy.fr

Citation: Diédhiou D, Weryha G, Angelousi A, Agopiantz M, Diop SN, et al. (2017) Impact of Parity on Fracture Risk After Menopause: A Systematic Review. J Hum Endocrinol 2: 009.

Received: July 27, 2016; Accepted: August 17, 2017; Published: August 31, 2017

Copyright: @ 2017 Diédhiou D, et al., This is an open-access article distributed under the terms of the Creative Commons Attribution License, which permits unrestricted use, distribution, and reproduction in any medium, provided the original author and source are credited. evaluated in 7 studies. One found a reduced risk in parous individuals $(\mathrm{HR}=0.71[0.52-0.97])$. An increased fracture risk was found in three studies. It's about Asian sub-population with $\geq 5$ children in two studies $(H R=1.65[1.06-2.56]$ and $R R=2.53[1.07-6.68])$. In another, a positive correlation between vertebral fracture and parity was reported $(\mathrm{OR}=1.093[1.008-1.186])$.

\section{Conclusion}

Overall, pregnancy does not seem to be associated with an increased risk of osteoporotic fracture after menopause. The negative impact of $\geq 5$ childbirth in Asiatic sub-population requires future investigations.

Keywords: Menopause; Parity; Risk fracture

\section{Abbreviations}

HR: Hazard Ratio; OR: Odds Ratio; HR: Hazard Ratio; CI: Confidence Interval; PTHrp: Parathyroid Hormone-Related Protein; BMD: Body Mass Density

\section{Introduction}

Osteoporosis is a disease essentially bound to menopause and ageing. The growing prevalence of osteoporosis is becoming an increasingly important health problem throughout the world. The estimations state a pandemic of osteoporosis in more than 200 million people in the world among which post-menopausal women represent $30 \%$ in United States and Europe. It expresses clinically after 50 years by fractures due to low energy traumas [1]. Osteoporotic fracture is due to the conjunction of minor trauma, lowered bone mass, change in trabecular bone microarchitecture and cortical porosity $[2,3]$. At least, $40 \%$ of osteoporotic women will suffer from one or several fragility fractures in their life [1].

Pregnancy and breastfeeding are periods of the woman's life with important calcic loss, estimated between 200 to $300 \mathrm{mg}$ daily [4]. This loss cause, in temporal way, a negative calcic balance which is stabilized by bone resorption [5]. Isolated observations of fragility fractures were especially reported in primipares. Their incidence remains underestimated and seems related to a preliminary fragility or an excessive bone resorption at the mother [6-9]. Albright and Reifenstein reported from 1948 the existence of osteoporotic fractures in two pregnant women [10].

The link between pregnancy, breastfeeding and osteoporotic fractures is not clear in the literature. The loss of bone mass during pregnancy and especially during breastfeeding varies from $1 \%$ per month to $10 \%$ during the 6 months of lactation. This phenomenon is however reversible during the weaning $[5,11,12]$. The accumulation of reproductive events during woman's life plays a very important role to clinical expression of osteoporotic disease. However, acquired and environmental factors as pregnancy and lactation seem to determinate the severity of osteoporotic disease [13].

For this purpose, we performed a systematic review in order to estimate the level of association between parity and risk of osteoporotic fracture after menopause.

\section{Materials and Methods}

\section{Data source and search strategy}

We conducted a review of the medical literature in order to identify 
all articles evaluating the association of parity and risk of post-menopausal fractures through a search of PubMed, Cochrane, Embase databases from January 1, 1980 through January 31, 2016 applying the following combined search keywords: (parity OR pregnancy OR lactation OR breastfeeding OR reproduction) AND (bone OR bone mineral density OR osteoporosis OR fracture). The bibliography of relevant articles was also searched. The search was restricted to English and French literature. Two reviewers (DD, GW) reviewed titles and abstracts to identify studies likely to report association between parity with fracture and subsequently obtained the full texts (Figure 1).

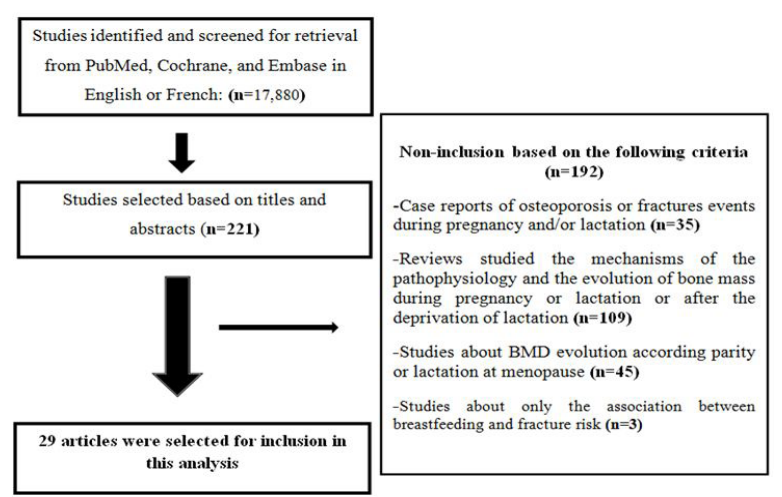

Figure 1: Studies selection process based on the inclusion and non-inclusion criteria.

\section{Study selection criteria}

To be eligible for inclusion in this systematic review, studies had to meet the following criteria:

I. Having a human prospective, retrospective, case control or cross sectional design;

II. Including a female population of premenopausal, menopausal or postmenopausal status;

III. All fractures diagnosis had to be based on clinical and/or imaging studies (radiography, bone densitometry, scanner);

IV. Having result of study evaluating the risk for one or overall fractures according to parity and expressed results with univariate and/or multivariate adjusted OR (Odds Ratio) or HR (Hazard Ratio) or RR (Relative Risk) with a Confidence Interval (CI) of 95\%, with or without $\mathrm{p}$ value.

We excluded papers reporting case reports, reviews, commentaries and letters, studies about mechanisms of Pathophysiology and evolution of bone mass during pregnancy or lactation or after deprivation of lactation as well as animal's experimental studies, studies about only Body Mass Density (BMD) evolution according to parity or lactation at menopause, studies about only the association between breastfeeding and fracture risk, studies in which the criteria of evaluation were not sufficient. Studies where fractures or low bone mass were due to hematological or endocrinological diseases (pex multiple myeloma, hyperparathyroidism, Cushing disease etc.,) or were medically induced (use of corticoids or...) were also excluded.

\section{Data extraction and quality assessment}

Two investigators (DD, AA) reviewed independently each eligible manuscript and extracted data on general characteristics of each study including first author's name, year of publication, study design, setting and follow up. We present data also as far as characteristics of studied population (sample size, age, and menopausal status when given) and compared groups as well as data concerning the studied outcome and the type of the statistical analysis (univariate and/or multivariate analysis) used for the extraction of the main outcomes. Studies were also organized regarding methodology and quality of proof. A label ranging from $\mathrm{A}$ to $\mathrm{C}$ was attached to each publication. According to French recommendation, a grade A is established on a scientific proof established by one studies of strong level of proof for example comparative tests randomized of strong power and without major bias, meta analysis of randomized controlled essays, analyzes of decision based on well led studies. A grade B is based on a scientific assumption supplied by studies of intermediate level of proof: for example, comparative tests randomized by low power, well led not randomized comparative studies, cohort studies. A recommendation of grade $\mathrm{C}$ is established on studies of lesser level of proof for example, case control studies, series of case [14]. When available, ethnic origins were taken in account.

\section{Results}

\section{Characteristics of the included studies}

A total of 17,880 studies were found through literature search. The majority of them $(17,659)$ were excluded based on title and abstract. From the 221 remaining studies, 29 studies were chosen to be evaluated in this systematic review based on full text (Figure 1).

Twenty nine studies presented data of association between parity and risk of fracture. Twelve studies included European population, eight studies North American population, one study South American population and two others a maghrebian population. Four studies were conducted in an Asiatic population and finally two studies in an Australian population. The follow up period varied from 1 to 29 years.

The risk of fracture was studied in 13 prospective, 7 transversal and 10 retrospective studies. Among these studies, 12 were grade B and 16 grade C. The publication of Petersen et al., [15] gives one part of prospective results (grade B) and one part of transversal results (grade C). It is about 17 studies examined the risk for hip's fracture, 8 the risk for vertebral's fracture, 7 the risk for wrist's fracture and 5 the risk for all fracture (Table 1 and Figure 2).

\section{Characteristics of the included population}

A total of 253,587 women were included in the 29 studies. It's about 6,149 (2.42\%) women at premenopausal status and 247,438 (97.58\%) at postmenopausal status. In this population, 52,375 women were Europeans (20.65\%), 190,225 North Americans (75.01\%), 1,855 South Americans (0.73\%), 6,092 Asians (2.4\%), 1,402 Australians (0.55\%) and 1,638 Maghrebian (0.64\%). It's about 221,632 parous, 30,013 nulliparous.

\section{Main results of studies (Figure 2)}

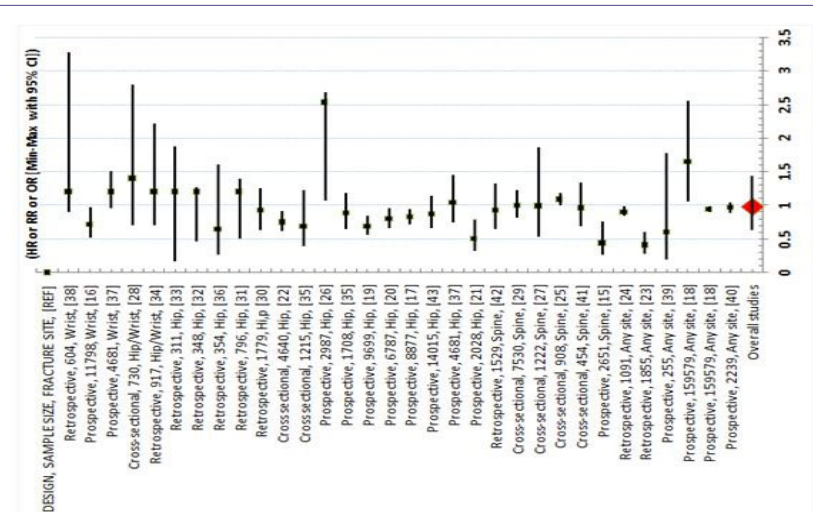

Figure 2: Synthesis of results about the association between parity and fracture risk in post-menopausal women.

Abbreviation: Min = Minimum; Max = Maximum; HR = Hazard Ratio; OR = Odds Ratio; $\mathrm{Cl}=$ Confidence Interval; REF = Reference 
Citation: Diédhiou D, Weryha G, Angelousi A, Agopiantz M, Diop SN, et al. (2017) Impact of Parity on Fracture Risk After Menopause: A Systematic Review. J Hum Endocrinol 2: 009 .

\begin{tabular}{|c|c|c|c|c|c|c|c|c|}
\hline $\begin{array}{l}\text { First author, } \\
\text { journal, year } \\
\text { of publication, } \\
\text { reference, } \\
\text { grade of rec- } \\
\text { ommendation }\end{array}$ & $\begin{array}{c}\text { Type of the studies, } \\
\text { setting }\end{array}$ & $\begin{array}{l}\text { Effect of } \\
\text { parity and } / \\
\text { or lacta- } \\
\text { tion on } \\
\text { fracture }\end{array}$ & $\begin{array}{c}\text { Sample size, } \\
\text { menopausal } \\
\text { status, age } \\
\text { in years } \\
\text { (mean } \pm \text { DS } \\
\text { or median) }\end{array}$ & $\begin{array}{l}\text { Fol- } \\
\text { low-up } \\
\text { (years) }\end{array}$ & $\begin{array}{l}\text { Compared } \\
\text { groups }\end{array}$ & Studied outcome & $\begin{array}{l}\text { Results: [incidence, } \\
\text { (HR or OR or RR } \\
\text { (95\% Cl), p value] }\end{array}$ & Conclusion \\
\hline $\begin{array}{l}\text { Mori et al., } \\
\text { Bone, [16] } \\
\text { Grade B }\end{array}$ & $\begin{array}{l}\text { Prospective, (The } \\
\text { Study of Women's } \\
\text { Health Across the } \\
\text { Nation), United-State }\end{array}$ & All fracture & $\begin{array}{l}2239,(1210 \\
\text { premeno- } \\
\text { pausal and } \\
1017 \text { post- } \\
\text { menopausal), } \\
(46.0 \pm 2.0)\end{array}$ & 15.7 & $\begin{array}{l}-2239 \text { parous } \\
\text { for } 1 \text { to } 3 \\
\text { children }\end{array}$ & $\begin{array}{l}\text { - Incidence of fractures } \\
\text { on the whole studied } \\
\text { population } \\
\text { - Adjusted }{ }^{1} \mathrm{HR} \text { in } \\
\text { compared groups for } \\
\text { fracture risk }\end{array}$ & $\begin{array}{l}-15.9 \% \\
\text { - Parity per additional } \\
\text { childbirth: } 0.97(0.89- \\
1.05), p=\text { nd }\end{array}$ & $\begin{array}{l}\text { Parity is not } \\
\text { associated } \\
\text { with fracture } \\
\text { risk }\end{array}$ \\
\hline $\begin{array}{l}\text { Cauley et } \\
\text { al., J Bone } \\
\text { Miner Res, [17] } \\
\text { Grade B }\end{array}$ & $\begin{array}{l}\text { Prospective, (Wom- } \\
\text { en's Health Initiative } \\
\text { study), United-State }\end{array}$ & All fracture & $\begin{array}{l}\text { 159579, post- } \\
\text { menopausal, } \\
(50-79)\end{array}$ & $8 \pm 2.6$ & $\begin{array}{c}144094 \\
\text { parous vs. } \\
15485 \text { nullipa- } \\
\text { rous* }^{*}\end{array}$ & $\begin{array}{c}\text { Adjusted }{ }^{2} \text { HR for } \\
\text { fractures risk across } \\
\text { ethnicity (White, Black, } \\
\text { Hispanic, Asian and } \\
\text { American Indian) }\end{array}$ & $\begin{array}{c}2-4 \text { children: } 0.94(0.90- \\
0.99), 1.10(0.82-1.46), \\
0.85(0.60-1.20), \\
1.25(0.84-1.87), \\
1.09(0.35-3.42), p=\text { nd } \\
\geq 5 \text { children: } 0.95(0.90- \\
1.00), 1.13(0.84-1.54), \\
0.81(0.56-1.16), \\
1.65(1.06-2.56), \\
1.43(0.44-4.61), p=\text { nd }\end{array}$ & $\begin{array}{c}\text { Parity is } \\
\text { associated to } \\
\text { a lower risk } \\
\text { for fracture in } \\
\text { white parous } \\
\text { with } 2-4 \\
\text { children and } \\
\text { a higher risk } \\
\text { for fracture in } \\
\text { asian parous } \\
\text { with } \geq 5 \text { chil- } \\
\text { dren }\end{array}$ \\
\hline $\begin{array}{l}\text { Paganini-Hill et } \\
\text { al., J Womens } \\
\text { Health, [18] } \\
\text { Grade B }\end{array}$ & $\begin{array}{l}\text { Prospective, (The } \\
\text { Leisure World } \\
\text { Cohort Study), } \\
\text { United-State }\end{array}$ & $\begin{array}{l}\text { Hip, wrist } \\
\text { and spine } \\
\text { fractures }\end{array}$ & $\begin{array}{l}8877, \text { post- } \\
\text { menopausal, } \\
(73 \pm 7.4)\end{array}$ & 20 & $\begin{array}{l}6549 \text { parous } \\
\text { vs. } 2308 \\
\text { nulliparous }\end{array}$ & $\begin{array}{l}\text { - Incidence of hip, } \\
\text { wrist, spine fracture } \\
\text { on whole studied } \\
\text { population } \\
\text { - Adjusted }{ }^{3} \mathrm{HR} \text { in } \\
\text { compared groups } \\
\text { for hip, wrist, spine } \\
\text { fracture risk }\end{array}$ & $\begin{array}{c}0.83(0.72-0.95) \\
0.91(n d), 0.98(n d), \\
p=n d\end{array}$ & $\begin{array}{l}\text { Parity is } \\
\text { associated to } \\
\text { a significant } \\
\text { lower risk for } \\
\text { hip fracture }\end{array}$ \\
\hline $\begin{array}{c}\text { Taylor et al., J } \\
\text { Am Geriatr Soc, } \\
\text { [19] Grade B }\end{array}$ & $\begin{array}{l}\text { Prospective, (Study } \\
\text { of Osteoporotic } \\
\text { Fractures), United } \\
\text { State }\end{array}$ & Hip fracture & $\begin{array}{l}6787, \text { post- } \\
\text { menopausal, } \\
(73.3 \pm 4.9)\end{array}$ & $10.1 \pm 3.2$ & $\begin{array}{l}5558 \text { parous* } \\
\text { vs. } 1229 \\
\text { nulliparous }\end{array}$ & $\begin{array}{c}\text { - Incidence of hip frac- } \\
\text { ture on whole studied } \\
\text { population } \\
\text {-Adjusted }{ }^{4} \mathrm{HR} \text { in } \\
\text { compared groups (with } \\
\text { and without BMD) for } \\
\text { hip fracture risk }\end{array}$ & $\begin{array}{c}8.9 \% \\
\text { With BMD: b1.28(1.06- } \\
1.55) \\
\text { Without BMD : } \\
\text { 1.25(1.04-1.51) p = nd }\end{array}$ & $\begin{array}{l}\text { Nulliparity is } \\
\text { associated to } \\
\text { a significant } \\
\text { higher risk for } \\
\text { hip fracture }\end{array}$ \\
\hline $\begin{array}{c}\text { Hillier et } \\
\text { al., J Bone } \\
\text { Miner Res, [20] } \\
\text { Grade B }\end{array}$ & $\begin{array}{l}\text { Prospective, (Study } \\
\text { of Osteoporotic } \\
\text { Fractures), Unit- } \\
\text { ed-State }\end{array}$ & $\begin{array}{l}\text { Non } \\
\text { traumatic } \\
\text { hip, spine, } \\
\text { and wrist } \\
\text { fractures }\end{array}$ & $\begin{array}{l}9699 \text {, post- } \\
\text { menopausal, } \\
(72.9 \pm 5.6 \text { for } \\
\text { nulliparous } \\
\text { and } 71 \pm 5.2 \\
\text { for parous) }\end{array}$ & 3 & $\begin{array}{l}7864 \text { parous }^{*} \\
\text { vs. } 1835 \\
\text { nulliparous }\end{array}$ & $\begin{array}{l}\text { - Incident of hip, spine } \\
\text { and wrist fracture } \\
\text { on whole studied } \\
\text { population } \\
\text { - Adjusted }{ }^{5} \mathrm{HR} \text { in } \\
\text { compared groups (with } \\
\text { and without BMD) for } \\
\text { hip, spine and wrist } \\
\text { fracture risk }\end{array}$ & $\begin{array}{c}6.1 \%, 4 \%, 6.5 \% \\
\text { With BMD : } 1.44(1.17- \\
1.78), 1.14(0.85-1.52) \\
\text { and } 0.86(0.69-1.09) \\
\text { Without BMD : } \\
1.44(1.17-1.78) \\
1.09(0.82-1.45) \text { and } \\
0.87(0.69-1.09), p=\text { nd }\end{array}$ & $\begin{array}{l}\text { Nulliparity is } \\
\text { associated to } \\
\text { a significant } \\
\text { higher risk for } \\
\text { hip fracture }\end{array}$ \\
\hline $\begin{array}{l}\text { Bjørnerem et } \\
\text { al., J Bone } \\
\text { Miner Res, [21] } \\
\text { Grade B }\end{array}$ & $\begin{array}{c}\text { Prospective, } \\
\text { (Tromsø Study), } \\
\text { Norway }\end{array}$ & $\begin{array}{l}\text { Hip and } \\
\text { wrist frac- } \\
\text { tures }\end{array}$ & $\begin{array}{l}4681 \text {, post- } \\
\text { menopausal, } \\
(63.9)\end{array}$ & 14.5 & $\begin{array}{l}\text { - } 4230 \text { parous } \\
\text { vs. } 451 \text { nullip- } \\
\text { arous* }\end{array}$ & $\begin{array}{c}\text { - Incidence of hip, fra- } \\
\text { gility and wrist fractures } \\
\text { on the whole studied } \\
\text { population } \\
\text { - Adjusted }{ }^{6} \mathrm{HR} \text { in com- } \\
\text { pared groups for hip } \\
\text { and wrist fracture risk }\end{array}$ & $\begin{array}{c}9.4 \%, 13.2 \% \\
1.04(0.75-1.46), p= \\
0.89 ; \text { and } 1.20(0.96- \\
1.51), p=0.80\end{array}$ & $\begin{array}{l}\text {-Parity is not } \\
\text { associated } \\
\text { to the risk for } \\
\text { hip and wrist } \\
\text { fracture }\end{array}$ \\
\hline $\begin{array}{l}\text { Kauppi et al., } \\
\text { Osteoporosis } \\
\text { Int, [22] } \\
\text { Grade B }\end{array}$ & $\begin{array}{c}\text { Prospective, } \\
\text { (Mini-Finland Health } \\
\text { Survey study), } \\
\text { Finland }\end{array}$ & Hip fracture & $\begin{array}{c}2028, \text { post- } \\
\text { menopausal, } \\
(63.2 \pm 9.2)\end{array}$ & 17 & $\begin{array}{l}1633 \text { parous } \\
\text { vs. } 395 \text { nullip- } \\
\text { arous }^{*}\end{array}$ & $\begin{array}{l}\text { - Incidence of hip } \\
\text { fracture on the whole } \\
\text { studied population } \\
\text { - Adjusted }{ }^{7} \text { RR in } \\
\text { compared groups for } \\
\text { hip fracture risk }\end{array}$ & $\begin{array}{c}6.5 \% \\
1-2 \text { children: } 0.85(0.55- \\
1.32) \\
\geq 3 \text { children: } 0.5(0.32- \\
0.79) \\
p=\text { nd }\end{array}$ & $\begin{array}{l}\text { Only parity } \\
\geq 3 \text { birth is } \\
\text { associated to } \\
\text { a significant } \\
\text { lower risk of } \\
\text { hip fracture }\end{array}$ \\
\hline $\begin{array}{l}\text { Trémollieres et } \\
\text { al., J Bone Min- } \\
\text { er Res, [23] } \\
\text { Grade B }\end{array}$ & $\begin{array}{l}\text { Prospective, (Meno- } \\
\text { pause et Os Cohort } \\
\text { Study), French }\end{array}$ & $\begin{array}{l}\text { Spine } \\
\text { and hip } \\
\text { fractures }\end{array}$ & $\begin{array}{l}2651,(756 \\
\text { premeno- } \\
\text { pausal and } \\
1895 \text { post- } \\
\text { menopausal, } \\
(54 \pm 4)\end{array}$ & 13.4 & $\begin{array}{l}2416 \text { parous } \\
\text { vs. } 235 \text { nullip- } \\
\text { arous* }\end{array}$ & $\begin{array}{c}\text {-Incidence of fracture } \\
\text { on the whole studied } \\
\text { population } \\
\text { - BMD adjusted }{ }^{8} \mathrm{HR} \text { in } \\
\text { compared groups for } \\
\text { spine and hip fracture } \\
\text { risk }\end{array}$ & $\begin{array}{c}15.6 \% \\
2 \text { children: } 0.68(0.42- \\
1.11) \text { and } 0.66(0.36- \\
1.22) \\
\geq 3 \text { children: } 0.44(0.26- \\
0.76) \text { and } 0.52(0.27- \\
1.00), p=\text { nd }\end{array}$ & $\begin{array}{l}\text { Only parity } \\
\geq 3 \text { birth is } \\
\text { associated to } \\
\text { a significant } \\
\text { lower risk for } \\
\text { spine fracture }\end{array}$ \\
\hline
\end{tabular}


Citation: Diédhiou D, Weryha G, Angelousi A, Agopiantz M, Diop SN, et al. (2017) Impact of Parity on Fracture Risk After Menopause: A Systematic Review. J Hum Endocrinol 2: 009.

\begin{tabular}{|c|c|c|c|c|c|c|c|c|}
\hline $\begin{array}{l}\text { Hundrup et al., } \\
\text { Eur J Epidemi- } \\
\text { ol, [24] } \\
\text { Grade B }\end{array}$ & $\begin{array}{l}\text { Prospective, Den- } \\
\text { mark, The Danish } \\
\text { Nurse Cohort Study }\end{array}$ & Hip fracture & $\begin{array}{l}14015 \text { post- } \\
\text { menopausal, } \\
(\geq 50)\end{array}$ & 6 & $\begin{array}{l}11120 \text { parous } \\
\text { vs } 2762 \\
\text { nulliparous* }\end{array}$ & $\begin{array}{c}\text { - Non adjusted HR in } \\
\text { compared groups for } \\
\text { hip fracture risk }\end{array}$ & $\begin{array}{c}0.87(0.66-1.15) \\
p=0.323\end{array}$ & $\begin{array}{l}\text { Parity is not } \\
\text { associated } \\
\text { to the risk for } \\
\text { hip and wrist } \\
\text { fracture }\end{array}$ \\
\hline $\begin{array}{c}\text { Naves et al., } \\
\text { Osteoporosis } \\
\text { Int, [25] } \\
\text { Grade B }\end{array}$ & $\begin{array}{c}\text { Prospective, } \\
\text { (European Vertebral } \\
\text { Osteoporosis Study), } \\
\text { Spain }\end{array}$ & $\begin{array}{l}\text { Vertebral } \\
\text { and no } \\
\text { vertebral } \\
\text { osteoporot- } \\
\text { ic fractures }\end{array}$ & $\begin{array}{l}255, \text { post- } \\
\text { menopausal, } \\
(65 \pm 9)\end{array}$ & 8 & $\begin{array}{c}-212 \text { Parous } \\
\text { vs. } 38 \text { nullipa- } \\
\text { rous* }^{*}\end{array}$ & $\begin{array}{l}\text { - Incident of osteo- } \\
\text { porotic fracture on } \\
\text { the whole studied } \\
\text { population } \\
\text { - Adjusted }{ }^{9} \text { OR in } \\
\text { compared groups for } \\
\text { osteoporotic fracture } \\
\text { risk }\end{array}$ & $\begin{array}{c}12.1 \% \\
0.60(0.20-1.78)\end{array}$ & $\begin{array}{l}\text { Parity is not } \\
\text { associated } \\
\text { with the risk for } \\
\text { osteoporotic } \\
\text { fracture }\end{array}$ \\
\hline $\begin{array}{c}\text { Petersen et al., } \\
\text { Ann Epidemiol, } \\
\text { [15] } \\
\text { Grade B }\end{array}$ & $\begin{array}{l}\text { Prospective, (The } \\
\text { Danish Twin Survey } \\
\text { Study), Denmark }\end{array}$ & Hip fracture & $\begin{array}{l}1708, \text { post- } \\
\text { menopausal, } \\
75 \text { (66-99) }\end{array}$ & 29.11 & $\begin{array}{l}-523 \text { parous } \\
1-2 \text { birth* vs. } \\
293 \text { nullipa- } \\
\text { rous and vs. } \\
352 \text { parous } \\
3-4 \text { birth }\end{array}$ & $\begin{array}{c}\text {-Incidence of hip frac- } \\
\text { ture on whole studied } \\
\text { population } \\
\text { - Non adjusted HR in } \\
\text { compared groups for } \\
\text { hip fracture risk }\end{array}$ & $\begin{array}{c}18.7 \% \\
\text { Nulliparity: } 1.28(0.98- \\
1.68) \\
3-4 \text { children: } 0.88(0.65- \\
1.19), p=\text { nd }\end{array}$ & $\begin{array}{l}\text { Parity is not } \\
\text { associated to } \\
\text { the risk for hip } \\
\text { fracture }\end{array}$ \\
\hline $\begin{array}{l}\text { Honkanen et } \\
\text { al., Osteoporo- } \\
\text { sis Int, [26] } \\
\text { Grade B }\end{array}$ & $\begin{array}{l}\text { Prospective, (Kuopio } \\
\text { Osteoporosis Risk } \\
\text { Factor and Preven- } \\
\text { tion Study), Finland }\end{array}$ & $\begin{array}{l}\text { Distal } \\
\text { forearm } \\
\text { fracture }\end{array}$ & $\begin{array}{l}11798,(3775 \\
\text { premeno- } \\
\text { pausal and } \\
8023 \text { post- } \\
\text { menopausal), } \\
(52.3 \pm 2.9)\end{array}$ & 5 & $\begin{array}{l}10488 \text { parous } \\
\text { vs. } 1310 \\
\text { nulliparous* }\end{array}$ & $\begin{array}{l}\text {-Incidence of forearm } \\
\text { fracture on whole } \\
\text { studied population } \\
\text { - Adjusted }{ }^{10} \mathrm{HR} \text { in com- } \\
\text { pared groups for distal } \\
\text { forearm fracture risk }\end{array}$ & $\begin{array}{c}3.1 \% \\
0.71(0.52-0.97) \\
p=0.031\end{array}$ & $\begin{array}{l}\text { Parity is } \\
\text { associated to } \\
\text { a significant } \\
\text { lower risk for } \\
\text { distal forearm } \\
\text { fracture }\end{array}$ \\
\hline $\begin{array}{l}\text { Fujiwara et al., } \\
\text { J Bone Miner } \\
\text { Res, [27] } \\
\text { Grade B }\end{array}$ & $\begin{array}{l}\text { Prospective, (Adult } \\
\text { Health Study), Japan }\end{array}$ & Hip fracture & $\begin{array}{c}2987 \text {, post- } \\
\text { menopausal, } \\
(58.6 \pm 11.6)\end{array}$ & 14 & $\begin{array}{l}1353 \text { parous } \\
1-2 \text { children* } \\
\text { vs. } 254 \text { nullip- } \\
\text { arous and vs. } \\
923 \text { parous } \\
3-4 \text { children } \\
\text { and vs. } 403 \\
\text { parous } \geq 5 \\
\text { children }\end{array}$ & $\begin{array}{l}\text { Adjusted }{ }^{11} \mathrm{RR} \text { in } \\
\text { compared groups for } \\
\text { hip fracture risk }\end{array}$ & $\begin{array}{c}\text { Nulliparity: } 2.31(0.60- \\
7.76) \\
\text { 3-4 children: } 1.26(0.51- \\
3.39) \\
\geq 5 \text { children: } 2.53(1.07- \\
6.68), p=\text { nd }\end{array}$ & $\begin{array}{l}\text { Parity } \geq 5 \\
\text { children is as- } \\
\text { sociated to a } \\
\text { higher risk for } \\
\text { hip fracture }\end{array}$ \\
\hline $\begin{array}{c}\text { Hwang et al., } \\
\text { Osteoporos Int, } \\
\text { [28] } \\
\text { Grade C }\end{array}$ & $\begin{array}{l}\text { Cross-sectional, (Ko- } \\
\text { rea National Health } \\
\text { and Nutrition } \\
\text { Examination Survey) } \\
\text { South Korea }\end{array}$ & $\begin{array}{l}\text { Vertebral } \\
\text { fracture }\end{array}$ & $\begin{array}{c}1222 \text { post- } \\
\text { menopausal, } \\
(62.8 \pm 8.8)\end{array}$ & 2 & $\begin{array}{c}444 \text { parous } \\
<3 \text { births* vs } \\
778 \text { parous } \\
\geq 3 \text { births }\end{array}$ & $\begin{array}{l}\text { Adjusted }{ }^{12} \text { OR in } \\
\text { compared groups for } \\
\text { hip fracture risk }\end{array}$ & $0.999(0.537-1.861)$ & $\begin{array}{l}\text { Parity is not } \\
\text { associated } \\
\text { with a risk } \\
\text { of vertebral } \\
\text { fracture }\end{array}$ \\
\hline $\begin{array}{l}\text { Lambrinoudaki } \\
\text { et al., Spine } \\
\text { J, [29] } \\
\text { Grade C }\end{array}$ & $\begin{array}{l}\text { Cross-sectional } \\
\text { study, Greece }\end{array}$ & $\begin{array}{l}\text { Vertebral } \\
\text { fracture }\end{array}$ & $\begin{array}{c}454, \text { post- } \\
\text { menopausal, } \\
(56.8 \pm 7.1)\end{array}$ & 5 & $\begin{array}{l}378 \text { parous } \\
\text { vs. } 68 \text { nullip- } \\
\text { arous }\end{array}$ & $\begin{array}{l}\text { Non adjusted OR in } \\
\text { compared groups for } \\
\text { vertebral fracture risk }\end{array}$ & $\begin{array}{l}\text { Parity per one extra } \\
\text { child : } 0.968(0.69- \\
1.34), p=0.847\end{array}$ & $\begin{array}{l}\text { Parity is not } \\
\text { associated } \\
\text { to the risk } \\
\text { for vertebral } \\
\text { fracture }\end{array}$ \\
\hline $\begin{array}{l}\text { Maghraoui et } \\
\text { al., Bone, [30] } \\
\text { Grade C }\end{array}$ & $\begin{array}{l}\text { Cross-sectional } \\
\text { study, Marocco }\end{array}$ & $\begin{array}{l}\text { Vertebral } \\
\text { fracture }\end{array}$ & $\begin{array}{l}908, \text { post- } \\
\text { menopausal, } \\
(60.9 \pm 7.7)\end{array}$ & 3 & $\begin{array}{l}\text { (nd) parous } \\
\text { vs. (nd) nullip- } \\
\text { arous* }\end{array}$ & $\begin{array}{c}\text { - Percentage of } \\
\text { vertebral fracture on } \\
\text { the whole studied } \\
\text { population } \\
\text { - Non adjusted OR in } \\
\text { compared groups for } \\
\text { vertebral fracture risk }\end{array}$ & $\begin{array}{c}42 \% \\
1.093(1.008-1.186) \\
p=0.031\end{array}$ & $\begin{array}{l}\text { Parity is } \\
\text { associated to } \\
\text { a significant } \\
\text { higher risk } \\
\text { for vertebral } \\
\text { fracture }\end{array}$ \\
\hline $\begin{array}{l}\text { Allali et al., } \\
\text { Maturitas, [31] } \\
\text { Grade C }\end{array}$ & $\begin{array}{l}\text { Cross-sectional, } \\
\text { Marocco }\end{array}$ & $\begin{array}{l}\text { Osteo- } \\
\text { porotic } \\
\text { peripheral } \\
\text { fracture }\end{array}$ & $\begin{array}{l}730 \text {, post- } \\
\text { menopausal, } \\
(59.4 \pm 7.6)\end{array}$ & nd & $\begin{array}{c}663 \text { parous } \\
\text { vs } 67 \text { nullipa- } \\
\text { rous* }\end{array}$ & $\begin{array}{l}\text { Adjusted }^{13} \text { OR in } \\
\text { compared groups for } \\
\text { osteoporotic peripheral } \\
\text { fracture risk }\end{array}$ & $\begin{array}{c}1-3 \text { children: } 1.40(0.70- \\
2.80), p=0.3 \\
4-5 \text { children: } 1.10(0.53- \\
2.28), p=0.7 \\
\geq 6 \text { children: } 0.85(0.39- \\
1.80), p=0.6\end{array}$ & $\begin{array}{l}\text { Parity is not } \\
\text { associated to } \\
\text { the risk for } \\
\text { peripheral } \\
\text { fracture }\end{array}$ \\
\hline $\begin{array}{c}\text { Petersen et al., } \\
\text { Ann Epidemiol, } \\
\text { [15] } \\
\text { Grade C }\end{array}$ & $\begin{array}{l}\text { Cross sectional, } \\
\text { (The Longitudinal } \\
\text { Study of Aging } \\
\text { Danish Twins 1995), } \\
\text { Denmark }\end{array}$ & Hip fracture & $\begin{array}{l}1215, \text { post- } \\
\text { menopausal, } \\
80 \text { (75-98) }\end{array}$ & 18 & $\begin{array}{l}-531 \text { parous } \\
1-2 \text { birth* vs. }^{*} \\
226 \text { nullipa- } \\
\text { rous and vs. } \\
355 \text { parous } \\
3-4 \text { birth }\end{array}$ & $\begin{array}{l}\text { - Percentage of hip } \\
\text { fracture on whole } \\
\text { studied population } \\
\text { - Non adjusted OR in } \\
\text { compared groups for } \\
\text { hip fracture risk }\end{array}$ & $\begin{array}{c}77.3 \% \\
\text { Nulliparity: } 1.18(0.69- \\
2.02) \\
\text { 3-4 children: } 0.69(0.39- \\
1.23), p=\text { nd }\end{array}$ & $\begin{array}{c}\text { Parity is not } \\
\text { associated to } \\
\text { the risk for hip } \\
\text { fracture }\end{array}$ \\
\hline $\begin{array}{l}\text { Michaelsson et } \\
\text { al., Am J Epide- } \\
\text { miol, [32] } \\
\text { Grade C }\end{array}$ & $\begin{array}{l}\text { Cross sectional, } \\
\text { case-control study, } \\
\text { Sweden }\end{array}$ & Hip fracture & $\begin{array}{c}4640, \text { post- } \\
\text { menopausal, } \\
(72.5 \pm 6.8 \text { in } \\
\text { cases and } \\
70.5 \pm 7.7 \text { in } \\
\text { controls) }\end{array}$ & 3 & $\begin{array}{l}-3848 \text { parous } \\
\text { vs. } 792 \text { nullip- } \\
\text { arous* }\end{array}$ & $\begin{array}{l}\text { - Percentage of hip } \\
\text { fracture on whole } \\
\text { studied population } \\
\text { - Adjusted }{ }^{14} \text { OR in } \\
\text { compared groups for } \\
\text { hip fracture risk }\end{array}$ & $\begin{array}{c}28.6 \% \\
1 \text { child: } 0.90(0.73- \\
1.12), \\
2 \text { children: } 0.75(0.62- \\
0.91) \\
3 \text { children: } 0.80(0.66- \\
0.98), p=\text { nd }\end{array}$ & $\begin{array}{l}\text { Parity } \geq 2 \\
\text { children is } \\
\text { protective for } \\
\text { hip fracture }\end{array}$ \\
\hline
\end{tabular}


Citation: Diédhiou D, Weryha G, Angelousi A, Agopiantz M, Diop SN, et al. (2017) Impact of Parity on Fracture Risk After Menopause: A Systematic Review. J Hum Endocrinol 2: 009 .

\begin{tabular}{|c|c|c|c|c|c|c|c|c|}
\hline $\begin{array}{c}\text { O'Neill et al., } \\
\text { Osteoporos Int, } \\
\text { [33] } \\
\text { Grade C }\end{array}$ & $\begin{array}{c}\text { Cross-sectional, } \\
\text { (European Vertebral } \\
\text { Osteoporosis Study), } \\
\text { United-Kingdom }\end{array}$ & $\begin{array}{l}\text { Vertebral } \\
\text { deformity }\end{array}$ & $\begin{array}{l}7530, \text { post- } \\
\text { menopausal, } \\
(67.3 \pm 7.9 \text { in } \\
\text { cases and } \\
62.6 \pm 7.9 \text { in } \\
\text { controls) }\end{array}$ & $\mathrm{Nd}$ & $\begin{array}{l}\text { - } 6398 \text { parous } \\
\text { vs. } 1132 \\
\text { nulliparous }\end{array}$ & $\begin{array}{l}\text { Adjusted }{ }^{15} \mathrm{OR} \text { in } \\
\text { compared groups for } \\
\text { vertebral deformity }\end{array}$ & $\begin{array}{c}1.00(0.82-1.23) \\
p=n d\end{array}$ & $\begin{array}{l}\text { Parity is not } \\
\text { associated } \\
\text { to the risk } \\
\text { for vertebral } \\
\text { deformity }\end{array}$ \\
\hline $\begin{array}{l}\text { Shin et al., J } \\
\text { Bone Miner } \\
\text { Metab, [34] } \\
\text { Grade C }\end{array}$ & $\begin{array}{c}\text { Retrospective, } \\
\text { (Ansung community } \\
\text { cohort study), Korea }\end{array}$ & $\begin{array}{l}\text { Vertebral } \\
\text { fracture }\end{array}$ & $\begin{array}{c}1529,(314 \\
\text { premeno- } \\
\text { pausal and } \\
1215 \text { post- } \\
\text { menopausal), } \\
(59.1 \pm 8.7)\end{array}$ & 2 & $\begin{array}{l}687 \text { parous } \\
\geq 4 \text { births vs. } \\
809 \text { parous } \\
<4 \text { births }{ }^{*}\end{array}$ & $\begin{array}{c}\text { - Percentage of } \\
\text { vertebral fracture on } \\
\text { the whole studied } \\
\text { population } \\
\text { - Age adjusted OR in } \\
\text { compared groups for } \\
\text { vertebral fracture risk }\end{array}$ & $\begin{array}{c}14.8 \% \\
0.93(0.65-1.32) \\
p=n d\end{array}$ & $\begin{array}{l}\text { Parity is not } \\
\text { associated } \\
\text { to the risk } \\
\text { for vertebral } \\
\text { fracture }\end{array}$ \\
\hline $\begin{array}{l}\text { Wengreen et } \\
\text { al., Osteoporos } \\
\text { Int, [35] } \\
\text { Grade C }\end{array}$ & $\begin{array}{c}\text { Retrospective, } \\
\text { Case control study, } \\
\text { (Utah Study of } \\
\text { Nutrition and Bone } \\
\text { Health), United State }\end{array}$ & Hip fracture & $\begin{array}{c}1779, \text { post- } \\
\text { menopausal, } \\
(76.7 \pm 9.1)\end{array}$ & 5 & $\begin{array}{l}1624 \text { parous } \\
\text { vs. } 155 \text { nullip- } \\
\text { arous* }^{*}\end{array}$ & $\begin{array}{l}\text { - Percentage of hip } \\
\text { fracture on whole } \\
\text { studied population } \\
\text { - Adjusted }{ }^{16} \text { OR in } \\
\text { compared groups for } \\
\text { hip fracture risk }\end{array}$ & $\begin{array}{c}49.5 \% \\
0.93(0.63-1.25) \\
p=n d\end{array}$ & $\begin{array}{c}\text { Parity is not } \\
\text { associated to } \\
\text { the risk for hip } \\
\text { fracture }\end{array}$ \\
\hline $\begin{array}{c}\text { Huo et al., } \\
\text { Osteoporos Int, } \\
\text { [36] } \\
\text { Grade C }\end{array}$ & $\begin{array}{c}\text { Retrospective, } \\
\text { Case control study, } \\
\text { China }\end{array}$ & Hip fracture & $\begin{array}{l}354, \text { post- } \\
\text { menopausal, } \\
(67.1 \pm 8.3)\end{array}$ & 2 & $\begin{array}{l}-115 \text { parous } \\
1-2 \text { birth }^{\star} \text { vs. } \\
83 \text { parous } \geq 5 \\
\text { birth and vs. } \\
18 \text { nulliparous }\end{array}$ & $\begin{array}{l}\text { - Percentage of hip } \\
\text { fracture on whole } \\
\text { studied population } \\
\text { - Adjusted }{ }^{17} \text { OR in } \\
\text { compared groups for } \\
\text { hip fracture risk }\end{array}$ & $\begin{array}{c}33.3 \% \\
\text { Nulliparity: } 0.79(0.19 \\
-3.25) \\
\geq 5 \text { children: } 0.64(0.26- \\
1.61), p=0.49\end{array}$ & $\begin{array}{l}\text { Parity is not } \\
\text { associated } \\
\text { to the risk of } \\
\text { fracture }\end{array}$ \\
\hline $\begin{array}{l}\text { Cure-Cure et } \\
\text { al., Int J Gynae- } \\
\text { col Obstet, [37] } \\
\text { Grade C }\end{array}$ & $\begin{array}{c}\text { Retrospective, } \\
\text { Columbia }\end{array}$ & All fracture & $\begin{array}{l}\text { 1855, post- } \\
\text { menopausal, } \\
(61.3 \pm-8.3)\end{array}$ & 5 & $\begin{array}{l}1612 \text { parous } \\
\text { vs. } 243 \text { nullip- } \\
\text { arous* }^{*}\end{array}$ & $\begin{array}{l}\text { - Percentage of frac- } \\
\text { ture on whole studied } \\
\text { population } \\
\text { - Non adjusted OR in } \\
\text { compared groups for } \\
\text { all fracture risk }\end{array}$ & $\begin{array}{c}22.9 \% \\
0.41(0.28-0.61) \\
p<0.000002\end{array}$ & $\begin{array}{l}\text { Parity is as- } \\
\text { sociated with } \\
\text { a significant } \\
\text { lower risk for } \\
\text { fracture }\end{array}$ \\
\hline $\begin{array}{l}\text { Parazzini et } \\
\text { al., J Epidemiol } \\
\text { Community } \\
\text { Health, [38] } \\
\text { Grade C }\end{array}$ & $\begin{array}{l}\text { Retrospective, } \\
\text { Cross sectional, } \\
\text { (Italian case control } \\
\text { study), Italia }\end{array}$ & Hip fracture & $\begin{array}{l}\text { 796, post- } \\
\text { menopausal, } \\
(66)\end{array}$ & 10 & $\begin{array}{l}632 \text { Parous } \\
\text { vs. } 164 \text { nullip- } \\
\text { arous* }\end{array}$ & $\begin{array}{l}\text { Adjusted }^{18} \mathrm{OR} \text { in } \\
\text { compared groups for } \\
\text { hip fracture risk }\end{array}$ & $\begin{array}{c}0.8(0.5-1.4) \\
p=0.22\end{array}$ & $\begin{array}{l}\text { Parity is not } \\
\text { associated to } \\
\text { a risk for hip } \\
\text { fracture }\end{array}$ \\
\hline $\begin{array}{l}\text { Hoffman et al., } \\
\text { Osteoporosis } \\
\text { Int, [39] } \\
\text { Grade C }\end{array}$ & $\begin{array}{l}\text { Retrospective, } \\
\text { case-control study, } \\
\text { United-State }\end{array}$ & Hip fracture & $\begin{array}{l}348, \text { post- } \\
\text { menopausal, } \\
(\geq 50)\end{array}$ & 3 & $\begin{array}{l}\text { - } 233 \text { parous } \\
\text { vs. } 115 \text { nullip- } \\
\text { arous* }\end{array}$ & $\begin{array}{l}\text { - Percentage of hip } \\
\text { fracture on whole } \\
\text { studied population } \\
\text { - Adjusted }{ }^{19} \text { OR in } \\
\text { compared groups for } \\
\text { hip fracture risk }\end{array}$ & $\begin{array}{c}50 \% \\
0.76(0.46-1.27) \\
p=n d\end{array}$ & $\begin{array}{c}\text { Parity is not } \\
\text { associated to } \\
\text { the risk for hip } \\
\text { fracture }\end{array}$ \\
\hline $\begin{array}{l}\text { Nguyen et al., J } \\
\text { Clin Endocrinol } \\
\text { Metab, [40] } \\
\text { Grade C }\end{array}$ & $\begin{array}{l}\text { Retrospective, } \\
\text { (Dubbo Osteopo- } \\
\text { rosis Epidemiology } \\
\text { Study), Australia }\end{array}$ & $\begin{array}{l}\text { Atraumatic } \\
\text { fracture }\end{array}$ & $\begin{array}{l}\text { 1091, post- } \\
\text { menopausal, } \\
(70 \pm 7.2)\end{array}$ & 5 & $\begin{array}{l}990 \text { parous* } \\
\text { vs. } 101 \text { nullip- } \\
\text { arous }\end{array}$ & $\begin{array}{l}\text { Adjusted }{ }^{20} \mathrm{OR} \text { in com- } \\
\text { pared groups for no } \\
\text { traumatic fracture risk }\end{array}$ & $\begin{array}{c}1.10(1.01-1.19) \\
p<0.01\end{array}$ & $\begin{array}{l}\text { Nulliparity is } \\
\text { associated to } \\
\text { a significant } \\
\text { higher risk for } \\
\text { atraumatic } \\
\text { fracture }\end{array}$ \\
\hline $\begin{array}{l}\text { Mallmin et al., } \\
\text { Osteoporosis } \\
\text { Int, [41] } \\
\text { Grade C }\end{array}$ & $\begin{array}{l}\text { Retrospective, } \\
\text { case-control study, } \\
\text { Sweden }\end{array}$ & $\begin{array}{l}\text { Distal } \\
\text { forearm } \\
\text { fracture }\end{array}$ & $\begin{array}{c}604,(94 \text { pre- } \\
\text { menopausal } \\
\text { and } 510 \text { post- } \\
\text { menopausal), } \\
(62.8 \pm 10.1)\end{array}$ & 1 & $\begin{array}{c}-515 \text { parous } \\
\text { vs. } 89 \text { nullipa- } \\
\text { rous }^{*}\end{array}$ & $\begin{array}{l}\text { Adjusted }{ }^{21} \mathrm{OR} \text { in com- } \\
\text { pared group for distal } \\
\text { forearm fracture }\end{array}$ & $\begin{array}{c}1.72(0.90-3.28) \\
p=n d\end{array}$ & $\begin{array}{l}\text {-Parity is not } \\
\text { associated } \\
\text { to the risk } \\
\text { for forearm } \\
\text { fracture }\end{array}$ \\
\hline $\begin{array}{c}\text { Cumming et al., } \\
\text { Int J Epidemiol, } \\
\text { [42] } \\
\text { Grade C }\end{array}$ & $\begin{array}{l}\text { Retrospective, } \\
\text { case-control study, } \\
\text { Australia }\end{array}$ & Hip fracture & $\begin{array}{l}\text { 311, post- } \\
\text { menopausal, } \\
(\geq 65)\end{array}$ & 2 & $\begin{array}{c}-246 \text { parous } \\
\text { vs. } 65 \text { nullipa- } \\
\text { rous* }\end{array}$ & $\begin{array}{l}\text { - Percentage of hip } \\
\text { fracture on whole } \\
\text { studied population } \\
\text { - Adjusted }{ }^{22} \text { OR in } \\
\text { compared groups for } \\
\text { hip fracture risk }\end{array}$ & $\begin{array}{c}55.9 \% \\
0.56(0.17-1.88) \\
p=\text { nd }\end{array}$ & $\begin{array}{c}\text { Parity is not } \\
\text { associated to } \\
\text { the risk for hip } \\
\text { fracture }\end{array}$ \\
\hline $\begin{array}{c}\text { Alderman et al., } \\
\text { Am J Epidemiol, } \\
\text { [43] } \\
\text { Grade C }\end{array}$ & $\begin{array}{l}\text { Retrospective, } \\
\text { case-control study, } \\
\text { United-State }\end{array}$ & $\begin{array}{l}\text { Hip and } \\
\text { forearm } \\
\text { fractures }\end{array}$ & $\begin{array}{l}917, \text { post- } \\
\text { menopausal, } \\
(50-74)\end{array}$ & 5 & $\begin{array}{c}-734 \text { parous } \\
\text { vs. } 183 \text { nullip- } \\
\text { arous* }\end{array}$ & $\begin{array}{c}\text { - Percentage of frac- } \\
\text { ture on whole studied } \\
\text { population } \\
\text { - Adjusted }{ }^{23} \text { OR in } \\
\text { compared groups } \\
\text { for hip and forearm } \\
\text { fracture risk }\end{array}$ & $\begin{array}{c}38.7 \% \\
1.2(0.7-2.22) \\
p=\text { nd }\end{array}$ & $\begin{array}{c}\text { Parity is not } \\
\text { associated to } \\
\text { the risk for hip } \\
\text { and forearm } \\
\text { fractures }\end{array}$ \\
\hline
\end{tabular}

Table 1: Synthesis of the data of the studies having estimated the association between parity and fracture risk in post-menopausal women.

Abbreviations: $\mathrm{HR}=$ Hazard Ratio; OR = Odds Ratio; RR = Relative Risk; nd = no data; BMD = Bone Mineral Density; BMD: Bone Mass Density 
Citation: Diédhiou D, Weryha G, Angelousi A, Agopiantz M, Diop SN, et al. (2017) Impact of Parity on Fracture Risk After Menopause: A Systematic Review. J Hum Endocrinol 2: 009 .

\section{* Table 1 legend}

${ }^{1}$ Adjusted for age, race/ethnicity, menopausal transition stage, body mass index, smoking status, smoking pack-years, alcohol consumption level, physical activity level, employment status, diabetes, hyperthyroidism, current use of supplementary calcium, current use of supplementary vitamin D, prior use of sex steroid hormones, prior use birth control pills, prior use of Depo-Provera injection, current or prior use of oral corticosteroids, current use of proton pump inhibitors, other bone-adverse medications, and study site.

${ }^{2}$ Adjusted for age, years since menopause, education, living with a partner, height, weight, caffeine intake, smoking, fracture history, parental fracture history, falls, current HT use (5yr), corticosteroid use ( $>2$ years), sedative/ anxiolytics use, arthritis, depression, health status, and parity.

${ }^{3}$ Adjusted for age and others variables: for hip fracture (history of fracture, BMI, diabetes, glaucoma, smoking, vitamin A supplement use, attitude and having been pregnant), for wrist fracture (history of fracture, BMI, heart attack, alcohol consumption, vitamin A supplement use, cola intake and hysterectomy), for spine fracture (history of fracture, BMI, blood pressure medication, nonprescription pain medication, smoking, exercise, and attitude.

${ }^{4}$ Adjusted for total hip BMD, age, any previous fracture since age 50, history of maternal hip fracture after age 50, Parkinson's disease, type II diabetes mellitus, lowest quartile for distant depth perception, BMI, height at age 25, walking speed, digit symbol test number completed.

${ }^{5} \mathrm{Adjusted}$ for age, weight, height, maternal history of hip fracture, fracture of any bone after age 50 , self-reported, on feet $\leq 4 \mathrm{~h} / \mathrm{day}$, uses arms to stand from chair, history of diabetes, current calcium intake, current estrogen use, low frequency contrast sensitivity, resting pulse rate, and the interactions of self-reported health with on feet $\leq 4 \mathrm{~h} /$ day

${ }^{6} \mathrm{HR}$ adjusted for age, height, BMI (body mass index), smoking, alcohol use, physical activity, history of diabetes and previous wrist or hip fracture, use of hormone replacement therapy, and length of education

${ }^{7}$ Adjusted for age, age at last menstrual period, level of education, BMI, vitamin D status, parity, alcohol consumption, smoking history, leisure time physical activity and self-rated health.

${ }^{8}$ Adjusted for age, BMI, use of bisphosphonates, raloxifene or ranelate (past and present), use of estroprogestinic preparations (past and present), serum 25OHD levels, and number of pregnancies and presence of densitometric osteoporosis.

${ }^{9}$ Adjustment by age, handgrip strength, femoral neck BMD, prevalent vertebral fracture and the history of falls in the follow-up.

${ }^{10} \mathrm{Adjusted}$ for age, postmenopausal, body mass index, dairy calcium intake, hormone replacement therapy during follow-up, Wrist fracture history, parity.

${ }^{11}$ Adjusted for age, BMI, milk intake, alcohol intake, menarche, parity.

${ }^{12}$ Adjusted for age, BMI, age at menarche, duration of menopause, systolic blood pressure, GFR, PTH, 25(OH)D3, oral contraceptive use, HTN, DM, physical activity, alcohol and smoking status, number of deliveries, and age at first and last delivery.

${ }^{13}$ Adjustment for age (OR, 1.06; 95\% Cl, 0.97-1.15), BMI (OR, 1.01; 95\% Cl, 0.99-1.03), age at menopause (OR, 0.96; 95\% Cl, 0.89-1.04), time since menopause: (OR, 0.94; 95\% Cl, 0.87-1.02), wearing veil (OR, 1.02; 95\% Cl, 0.69-1.53) and total femoral BMD (OR, 0.13; 95\% Cl, 0.038-0.4).

${ }^{14}$ Adjusted for age $(=54,55-59,60-64,65-69,70-74$, and $=75$ years), hormone replacement therapy (never, former, and current use), oral contraceptive use (never and ever use), and body mass index (by quintiles)

${ }^{15}$ Adjusted for center, age, body mass index and smoking.

${ }^{16}$ Adjusted for age, education, BMI, history of estrogen use, age at menopause, history of oral contraceptive use, history of endometriosis, smoking status, vitamin $\mathrm{D}$ receptor genotype, lifetime physical activity, diabetes status, and history of breastfeeding.

${ }^{17}$ Adjusted for age, height, education, BMI, years lived in rural area, occupation, standing activities prior retirement, dietary calcium intake, breastfeeding and parity ${ }^{18}$ Adjusted for age, education, BMI, smoking status and estrogen replacement therapy.

${ }^{19}$ Adjusted for hospital of recruitment, age group, and age and body mass index.

${ }^{20}$ Adjusted for age, weight parity, estrogen exposure, hysterectomy.

${ }^{21}$ Adjusted for BMI, education, daily physical activity, leisure time activity, smoking, nulliparity, duration of HRT, menopausal discomfort, age at menopause.

${ }^{22}$ Adjustment for age, Body mass index, history of hormone replacement therapy use, current use of psychotropic medications, current smoking status, current dairy product consumption, score on mental state questionnaire, current physical activity and health status (number of self-report illnesses)

${ }^{23}$ Adjusted for attained for age, country and occupational group.

Association between parity and fracture risk: Ten studies found that multiparity had a protective role against the risk for fracture (IC 95\% variation of $\mathrm{RR}=[0.32-0.79], \mathrm{HR}=[0.26-0.99]$ and $\mathrm{OR}=$ [0.28-0.99]). Seven of them were prospective $[17-20,22,23,26]$ with a follow up between 3-20 years, one cross sectional [32] and two retrospective $[37,40]$. These results were found by multivariate adjusted analysis in eight studies [18-20,22,26,32,40]. Only three studies found a significant increased risk for vertebral fracture in Moroccan women $(\mathrm{OR}=1.09$, IC 95\% [1.00-1.18], $\mathrm{p}=0.031)$, [30] all fracture in Asian's North Americans parous with five or more children $(\mathrm{HR}=1.65$, IC 95\% $=[1.06-2.56])[17]$ and hip fracture in Japanese parous with five or more children $(\mathrm{RR}=2.53$, IC 95\% $=[1.07-6.68])$
[27]. In one study [17] the multivariate adjusted risk for osteoporotic fractures was lower in white women with $2-4$ children $(\mathrm{HR}=0.94$, IC 95\% $=[0.90-0.99])$, and higher in Asian's North American women with 5 or more children $(\mathrm{HR}=1.65$, IC $95 \%=[1.06-2.56])$ compared to nulliparous. The rest of 16 studies [15,16,21,24,25,28,29,31,33$36,38,39,41,42]$ found no statistical association between fracture and parity (Table 1 and Figure 2). It is necessary to note that no study on the American, European and Australian populations found a negative effect of the parity on the bone after the menopause. The only studies having found a higher risk for fracture resulted from an evaluation on the Asiatic or North African populations [17,27,30] (Table 2). 
Citation: Diédhiou D, Weryha G, Angelousi A, Agopiantz M, Diop SN, et al. (2017) Impact of Parity on Fracture Risk After Menopause: A Systematic Review. J Hum Endocrinol 2: 009.

- Page 7 of 10 •

\begin{tabular}{|c|c|c|c|}
\hline Population & References & Type of studies, Main criteria, simple size & Results: [incidence, (HR or OR or RR with $95 \% \mathrm{Cl})$ ] \\
\hline \multirow{8}{*}{ North American } & Mori et al. [16] & Prospective, Parity and all fracture, 2239 & $H R=0.97(0.89-1.05)$ \\
\hline & Cauley et al. [17] & Prospective, Parity and all fracture, 159579 & $\begin{array}{l}\text { White }(2-4 \text { children }): H R=0.94(0.90-0.99) \\
\text { Asian }(\geq 5 \text { children }): H R=1.65(1.06-2.56)\end{array}$ \\
\hline & Wengreen et al. [35] & Retrospective, Parity and hip fracture, 1779 & $\mathrm{OR}=0.93(0.63-1.25)$ \\
\hline & Paganini-Hill et al. [18] & Prospective, Parity and hip fracture, 8877 & $H R=0.83(0.72-0.95)$ \\
\hline & Taylor et al. [19] & Prospective, Parity and hip fracture, 6787 & $H R=0.80(0.66-0.96)$ \\
\hline & Hillier et al. [20] & Prospective, Parity and hip fracture, 9699 & $H R=0.69(0.56-0.85)$ \\
\hline & Hoffman et al. [39] & Retrospective, Parity and hip fracture, 348 & $\mathrm{OR}=0.76(0.46-1.27)$ \\
\hline & Alderman et al. [43] & Retrospective, Parity and hip or wrist fracture, 917 & $\mathrm{OR}=1.20(0.70-2.22)$ \\
\hline \multirow{13}{*}{ European } & Lambrinoudaki et al. [29] & Cross-sectional, Parity and spine fracture, 454 & $\mathrm{OR}=0.96(0.69-1.34)$ \\
\hline & Kauppi et al. [22] & Prospective, Parity $\geq 3$ children and hip fracture, 2028 & $R R=0.50(0.32-0.79)$ \\
\hline & Bjørnerem et al. [21] & Prospective, Parity and (hip, wrist) fracture, 4681 & $\begin{array}{l}\mathrm{HR}=1.04(0.75-1.46) \\
\mathrm{HR}=1.20(0.96-1.51)\end{array}$ \\
\hline & Trémollières et al. [23] & Prospective, Parity $\geq 3$ children and spine fracture, 2651 & $H R=0.44(0.26-0.76)$ \\
\hline & Hundrup et al. [24] & Prospective, Parity and hip fracture, 14015 & $\mathrm{HR}=0.87(0.66-1.15)$ \\
\hline & Naves et al. [25] & Prospective, Parity and osteoporotic fracture, 255 & $\mathrm{OR}=0.60(0.20-1.78)$ \\
\hline & Petersen et al. [15] & Prospective, Parity and hip fracture, 1708 & $H R=0.88(0.65-1.19)$ \\
\hline & Petersen et al. [15] & Cross sectional, Parity and hip fracture, 1215 & $\mathrm{OR}=0.69(0.39-1.23)$ \\
\hline & Michaelsson et al. [32] & Cross sectional, Parity $\geq 2$ children and hip fracture, 4640 & $\mathrm{OR}=0.75(0.62-0.91)$ \\
\hline & Honkanen et al. [26] & Prospective, Parity and wrist fracture, 11798 & $\mathrm{HR}=0.71(0.52-0.97)$ \\
\hline & O'Neill et al. [33] & Cross-sectional, Parity and spine fracture, 7530 & $\mathrm{OR}=1.00(0.82-1.23)$ \\
\hline & Parazzini et al. [38] & Retrospective, Parity and hip fracture, 796 & $\mathrm{OR}=0.80(0.50-1.40)$ \\
\hline & Mallmin et al. [41] & Retrospective, Parity and wrist fracture, 604 & $\mathrm{OR}=1.72(0.90-3.28)$ \\
\hline \multirow{4}{*}{ Asiatic } & Hwang et al. [28] & Cross-sectional, Parity $\geq 3$ children and spine fracture, 1222 & $\mathrm{OR}=0.99(0.53-1.86)$ \\
\hline & Shin et al. [34] & Retrospective, Parity and spine fracture, 1529 & $\mathrm{OR}=0.93(0.65-1.32)$ \\
\hline & Huo et al. [36] & Retrospective, Parity and all fracture, 354 & $\mathrm{OR}=0.64(0.26-1.61)$ \\
\hline & Fujiwara et al. [27] & Prospective, Parity $\geq 5$ children and hip fracture, 2987 & $R R=2.53(1.07-6.68)$ \\
\hline \multirow{2}{*}{ Maghrebian } & Maghraoui et al. [30] & Cross-sectional, Parity and spine fracture, 908 & $\mathrm{OR}=1.09(1.008-1.18)$ \\
\hline & Allali et al. [31] & Cross-sectional, Parity and peripheral fracture, 730 & $\mathrm{OR}=1.40(0.70-2.80)$ \\
\hline South American & Cure-Cure et al. [37] & Retrospective, Parity and all fracture, 1855 & $\mathrm{OR}=0.41(0.28-0.61)$ \\
\hline \multirow{2}{*}{ Australian } & Nguyen et al. [40] & Retrospective, Parity and all fracture, 1091 & $\mathrm{OR}=0.90(0.84-0.99)$ \\
\hline & Cumming et al. [42] & Retrospective, Parity and hip fracture, 311 & $\mathrm{OR}=0.56(0.17-1.88)$ \\
\hline
\end{tabular}

Table 2: Association of fracture risk and parity according the ethnicity.

Abbreviation: HR = Hazard Ratio; OR = Odds Ratio; RR = Relative Risk

Association between parity and hip fracture risk: Data concerning hip fracture risk were included in 17 studies [15,18$24,27,31,32,35,36,38,39,42,43]$. Five studies [18-20,22,32] found a statistically significant reduced risk for hip fracture in parous compared to nulliparous (IC 95\% variation of $\mathrm{RR}=[0.32-0.79], \mathrm{HR}=[0.56-0.96]$ and $\mathrm{OR}=[0.62-0.98])$. This result was confirmed by multivariate analysis in all studies. This reduced risk concerned especially women with two or more children in two studies $[22,32]$ with an OR $=0.75[0.62$ $0.91]$ and $R R=0.5[0.32-0.79]$. However, an increased risk for hip fracture was found in one prospective study concerned women with five or more children in Japanese postmenopausal population [27].

Association between parity and vertebral fracture risk: Eight studies evaluated the association of vertebral fracture to parity $[16,18,20,28-30,33,34]$. One of them [23] found a significant reduced risk for vertebral fracture in French women with three or more children $(\mathrm{OR}=0.44[0.26-0.76])$. However, one study found a significant increase risk for vertebral fracture in post-menopausal Moroccan parous population with an $\mathrm{OR}=1.093$ [1.008-1.186] [30].
Association between parity and wrist fracture risk: Seven studies examined the risk for wrist fracture $[18,20,21,26,31,41,43]$. One of these studies [26] found that the risk for wrist fracture was reduced in parous compared to nulliparous (IC 95\% variation of HR $=71[0.52$ 0.97]). This result was confirmed by a multivariate analysis.

\section{Discussion}

The impact of pregnancy on the risk of osteoporotic fracture in postmenopausal women is controversial. This systematic review analyzed the results of 29 selected clinical studies concerning the relationship between the risk of osteoporotic fracture at menopause and parity during the reproductive period of women.

Only three studies, two prospectives $[17,27]$ and one observational [30] associated parity to an increased risk of osteoporotic fracture after menopause. They involved, respectively, Asian-American, Japanese and North African populations. In all three studies, trabecular and cortical bone compartments were affected. The risk of fracture appeared in multivariate analysis to be associated with parity 5 or more 
children in the two prospective series with, respectively, $\mathrm{HR}=$ $1.65[1.06-2.56][18]$ and $\mathrm{RR}=2.53[1.07-6.68]$ [27]. This increased risk should be compared with the decrease in bone mass density, because fracture risk doubles for each point reduction in T-score [44]. Early pregnancy, its impact on the acquisition of peak bone mass $[45,46]$, poor contraceptive use and the impact of ethnic factors and/or nutritional habits (daily calcic ration) can be discussed. This negative link between parity and the fracture risk is limited to Asian women $[17,27]$. In all others studies in this population, even if not negative, now beneficial link is described $[28,34,36]$. At least, the Moroccan study show a negative link between parity and fracture risk, but exhibit methodological pitfalls: lack of control group, primary objective limited to worsening of vertebral fracture and no evaluation of osteoporotic fracture incidence [30]. In none Asiatic women, no data were support the hypothesis of a negative relationship between multiparity and fracture risk.

Ten out of the 29 studies reported a decrease in fracture risk in menopausal women that was attributable to parity. The majority of these included a Caucasian population. The risk of vertebral and non-vertebral fractures was reduced by $6 \%$ to $50 \%$ among menopausal parous compared with nulliparous women [18-20,26,37,40]. This reduced risk appears only beyond 2 to 3 pregnancies in four studies $[17,22,23,32]$. A reduction in the risk of vertebral fracture more than $50 \%(\mathrm{HR}=0.44[0.26-0.76])$ was reported by Trémollieres et al. [23]. In case-control studies, Michaelsson et al., [32] and Hillier et al., [20] found a reduced risk of hip fracture from $8 \%$ to $10 \%$ per child in postmenopausal women. The impact on the risk of wrist fracture was specifically assessed by Honkanen et al., [26] in a large prospective study of 11798 pre and postmenopausal women. The risk of wrist fracture in multivariate analysis was reduced by $30 \%$ ( $\mathrm{HR}=0.71[0.52$ 0.97]) among women with parity compared with nulliparous. The meta-analysis of Wang et al., [47] has included 10 prospective studies about parity and fracture in postmenopausal women. This paper is in accordance with our conclusion as it reports that increasing number of parity is associated with linearly reduced hip fracture risks among women. The osteoporosis fracture and hip fracture risks of parous women with at least one live birth were reduced by $11 \%$ and $26 \%$ respectively. The risk reduction for hip fracture was $12 \%$ for each one increased live birth.

These beneficial effects on all bone compartments are partly explicable by pathophysiological mechanisms and mechanical changes in bone structure. Bone loss during pregnancy and lactation are associated with hypersecretion of Parathyroid Hormone-Related Protein (PTHrp) and a fall in estrogen impregnation [48-50]. Increasing parity leads to functionally iterative estrogen deficiency. This promotes increased bone diameter by increasing periosteal apposition and endosteal resorption [51-54]. Mechanical resistance in torsion and flexion of a hollow cylinder increases exponentially with its diameter when the amount of material remains constant. This applies to the diaphyseal bone that undergoes a beneficial transformation during pregnancy, with no change in bone mass density or a slight decrease $[53,55]$.

However, conclusions from the included studies should be examined carefully because of important heterogeneity in studies. Studies differed considerably in their sampling profiles, follow-up times, and the extent of exposure to the risk of fracture. Regardless of the bone site and the population studied, the problem of reproducibility is addressed most often in retrospective studies data. In generally data in this synthesis of the literature suggest that multiparity do not generally appear to be deleterious to bone during the menopause.
The results of these studies provide no evidence for an association between pregnancy and an increased risk of osteoporotic fracture. Referring to the aggregate risk without stratification according to the number of pregnancies, the majority of studies in this systematic review agree that there is no significant influence of parity on fracture risk after the menopause either by population or bone site $[15,16,21,24,25,28,29,31,33-36,38,39,41,42]$. In agreement to these results, Henderson et al., [56] reported that postmenopausal osteoporotic risk is not affected by the occurrence of multiple pregnancies during lactation in multiparous women with more than five children. Our results confirm also the findings of Karlsson et al., [11] who reported a neutral or beneficial effect of pregnancy on osteoporotic risk after the menopause. A doubt remains for Asian women in whom osteoporotic risk increase as been described for five pregnancies or more. Additional studies are needed to clarify this point.

\section{Conclusion}

According to this review, pregnancy does not seem to be associated with an increased risk of osteoporotic fracture after menopause. Most of the studies agree on the absence of a significant effect, regardless of the population and the bone site. However, a non-negligible number of studies (especially Americans, Europeans and Australians) report a protective effect in bone by increased bone diameter due to increased periosteal apposition and endosteal resorption. Contraceptive use and the impact of ethnic factors and/or nutritional habits (daily calcic ration) could also be discussed in the studies on Asiatic or African population where a negative impact was reported. According to our analysis, the types of sample chosen and the study designs used explain at least partly these differences. Other powerful and rigorous studies are required to elucidate the link between parity and osteoporotic risk especially in Asian population. They will provide a basis on which to recommend preventive practices.

\section{Declaration}

\section{Authors' contributions}

DD, MA, CB and MLS carried out research and drafted the manuscript. DD, GW, OM, SND and MLS conceived of the study and participated in its design and coordination and helped to draft the manuscript. All authors read and approved the final manuscript.

\section{Competing interests}

The authors declare that they have no competing interests.

\section{References}

1. Melton LJ 3rd, Chrischilles EA, Cooper C, Lane AW, Riggs BL (1992) Perspective. How many women have osteoporosis?. J Bone Miner Res 7: 1005-1010

2. Silva MJ, Gibson LJ (1997) Modeling the mechanical behavior of vertebral trabecular bone: effects of age-related changes in microstructure. Bone 21: 191-199.

3. Currey JD (1999) What determines the bending strength of compact bone?. J Exp Biol 202: 2495-2503.

4. Prentice A (2000) Calcium in pregnancy and lactation. Annu Rev Nutr 20: $249-272$.

5. Ensom MH, Liu PY, Stephenson MD (2002) Effect of pregnancy on bone mineral density in healthy women. Obstet Gynecol Surv 57: 99-111.

6. Black AJ, Reid R, Reid DM, MacDonald AG, Fraser WD (2003) Effect of pregnancy on bone mineral density and biochemical markers of bone turnover in a patient with juvenile idiopathic osteoporosis. Bone Mineral Research 18: 167-171. 
Citation: Diédhiou D, Weryha G, Angelousi A, Agopiantz M, Diop SN, et al. (2017) Impact of Parity on Fracture Risk After Menopause: A Systematic Review. J Hum Endocrinol 2: 009

7. Peris P, Guañabens N, Monegal A, Pons F, Martínez De Osaba MJ, et al. (2002) Pregnancy associated osteoporosis: the familial effect. Clin Exp Rheumatol 20: 697-700.

8. Anai T, Tomiyasu T, Arima K, Miyakawa I (1999) Pregnancy-associated osteoporosis with elevated levels of circulating parathyroid hormone-related protein: a report of two cases. J Obstet Gynaecol Res 25: 63-67.

9. Timsit MA (2005) Bone demineralization and osteoporosis of pregnancy. Rev Rhum 72: 725-732.

10. Albright F, Reifenstein EC (1948) The Parathyroid Glands and Metabolic Bone Disease. Journal of Bone \& Joint Surgery 31: 881-882.

11. Karlsson MK, Ahlborg HG, Karlsson C (2005) Female reproductive history and the skeleton-a review. BJOG 112: 851-856.

12. Bezerra FF, Mendonça LM, Lobato EC, O’Brien KO, Donangelo CM (2004) Bone mass is recovered from lactation to postweaning in adolescent mothers with low calcium intakes. Am J Clin Nutr 80: 1322-1326.

13. Kovacs CS and Kronenberg HM (1997) Maternal-fetal calcium and bone metabolism during pregnancy, puerperium, and lactation. Endocr Rev 18: 832-872.

14. Laboratoire d'Enseignement et de Recherche sur le Traitement de l'Information Médicale (2000) Guide d'analyse de la littérature et gradation des recommandations. ANAES, Paris, France.

15. Petersen HC, Jeune B, Vaupel JW, Christensen K (2002) Reproduction life history and hip fractures. Annals Epidemiology 12: 257-263.

16. Tremollieres FA, Pouilles JM, Drewniak N, Laparra J, Ribot CA, et al. (2010) Fracture risk prediction using BMD and clinical risk factors in early postmenopausal women: sensitivity of the WHO FRAX Tool. J Bone Miner Res 25: 1002-1009.

17. Honkanen RJ, Honkanen K, Kröger H, Alhava E, Tuppurainen M, et al. (2000) Risk factors for perimenopausal distal forearm fracture. Osteoporos Int 11: 265-270.

18. Paganini-Hill A, Atchison KA, Gornbein JA, Nattiv A, Service SK, et al. (2005) Menstrual and reproductive factors and fracture risk: the Leisure World Cohort Study. J Womens Health 14: 808-819.

19. Cauley JA, Wu LL, Wampler NS, Barnhart JM, Allison M, et al. (2007) Clinical risk factors for fractures in multi-ethnic women: The Women's Health Initiative. J Bone Miner Res 22: 1816-1826.

20. Hillier TA, Rizzo JH, Pedula KL, Stone KL, Cauley JA, et al. (2003) Nulliparity and fracture risk in older women: The Study of Osteoporotic Fractures. J Bone Miner Res 18: 893-899.

21. Taylor BC, Schreiner PJ, Stone KL, Fink HA, Cummings SR, et al. (2004) Long-term prediction of incident hip fracture risk in elderly white women: Study of osteoporotic fractures. J Am Geriatr Soc 52: 1479-1486.

22. Kauppi M, Heliövaara M, Impivaara O, Knekt P, Jula A (2011) Parity and risk of hip fracture in postmenopausal women. Osteoporos Int 22: 1765 1771 .

23. Michaëlsson K, Baron JA, Farahmand BY, Ljunghall S (2001) Influence of parity and lactation on hip fracture risk. Am J Epidemiol 153: 1166-1172.

24. Cure-Cure C, Cure-Ramírez P, Terán E, López-Jaramillo P (2002) Bonemass peak in multiparity and reduced risk of bone-fractures in menopause. Int J Gynaecol Obstet 76: 285-291.

25. Nguyen TV, Jones G, Sambrook PN, White CP, Kelly PJ, et al. (1995) Ef fects of estrogen exposure and reproductive factors on bone mineral density and osteoporotic fractures. J Clin Endocrinol Metab 80: 2709-2714.

26. El Maghraoui A, Rezqi A, Mounach A, Achemlal L, Bezza A, et al. (2013) Systematic vertebral fracture assessment in symptomatic postmenopausal women. Bone 52: 176-180.
27. Fujiwara S, Kasagi F, Yamada M, Kodama K (1997) Risk factors for hip fracture in Japanese Cohort. J Bone Miner Res 12: 998-1004.

28. Hwang IR, Choi YK, Lee WK, Kim JG, Lee IK, et al. (2016) Association between prolonged breastfeeding and bone mineral density and osteoporosis in postmenopausal women: KNHANES 2010-2011. Osteoporos Int 27: 257-265.

29. Allali F, Maaroufi H, Aichaoui SE, Khazani H, Saoud B, et al. (2007) Influence of parity on bone mineral density and peripheral fracture risk in Moroccan postmenopausal women. Maturitas 57: 392-398.

30. O’Neill TW, Silman AJ, Diaz MN, Cooper C, Kanis J, et al. (1997) In fluence of hormonal and reproductive factors on the risk of vertebral deformity in European women. European Vertebral Osteoporosis Study Group. Osteoporos Int 7: 72-78.

31. Wengreen H, Cutler DR, Munger R, Willing M (2006) Vitamin D receptor genotype and risk of osteoporotic hip fracture in elderly women of Utah: an effect modified by parity. Osteoporosis International 17: 11461153.

32. Parazzini F, Bidoli E, Franceschi S, Schinella D, Tesio F, et al. (1996) Menopause, menstrual and reproductive history, and bone density in northern Italy. J Epidemiol Community Health 50: 519-523.

33. Hoffman S, Grisso JA, Kelsey JL, Gammon MD, and O’Brien LA (1993) Parity, lactation and hip fracture. Osteoporos Int 3: 171-176.

34. Cumming RG, Klineberg RJ (1993) Breastfeeding and other reproductive factors and the risk of hip fractures in elderly women. Int J Epidemiol 22: 684-691.

35. Alderman BW, Weiss NS, Daling JR, Ure CL, Ballard JH (1986) Reproductive history and postmenopausal risk of hip and forearm fracture. Am J Epidemiol 124: 262-277.

36. Huo D, Lauderdale DS, Li L (2003) Influence of reproductive factors on hip fracture risk in Chinese women. Osteoporos Int 14: 694-700.

37. Bjørnerem A, Ahmed LA, Jørgensen L, Størmer J, Joakimsen RM (2011) Breastfeeding protects against hip fracture in postmenopausal women: The Tromsø Study. J Bone Miner Res 26: 2843-2850.

38. Mallmin HS. Ljunghall S, Persson I, and Bergström R (1994) Risk fac tors for fractures of the distal forearm: A population-based case-control study. Osteoporos Int 4: 298-304.

39. Naves M, Diaz-Lopez JB, Gomez C, Rodriguez-Rebollar A, Cannata-An dia JB (2005) Determinants of incidence of osteoporotic fractures in the female Spanish population older than 50. Osteoporos Int 16: 2013-2017.

40. Mori T, Ishii S, Greendale GA, Cauley JA, Ruppert K, et al. (2015) Parity, lactation, bone strength, and 16-year fracture risk in adult women: Findings from the Study of Women's Health Across the Nation (SWAN). Bone 73: 160-166.

41. Lambrinoudaki I, Flokatoula M, Armeni E, Pliatsika P, Augoulea A, et al. (2015) Vertebral fracture prevalence among Greek healthy middle-aged postmenopausal women: association with demographics, anthropometric parameters, and bone mineral density. Spine J 15: 86-94.

42. Shin CS, Kim MJ, Shim SM, Kim JT, Yu SH, et al. (2012) The prevalence and risk factors of vertebral fractures in Korea. J Bone Miner Metab 30: 183-192.

43. Hundrup YA, Ekholm O, Høidrup S, Davidsen M, Obel EB (2005) Risk factors for hip fracture and a possible effect modification by hormone replacement therapy. The Danish nurse cohort study. Eur J Epidemiol 20: 871-877.

44. Marshall D, Johnell O, Wedel H (1996) Meta-analysis of how well measures of bone mineral density predict occurrence of osteoporotic fractures. BMJ 312: 1254.

45. Cho GJ, Shin JH, Yi KW, Park HT, Kim T, et al. (2012) Adolescent preg nancy is associated with osteoporosis in postmenopausal women. Menopause 19: 456-460. 
46. Schnatz PF, Barker KG, Marakovits KA, O'Sullivan DM (2010) Effects of age at first pregnancy and breast-feeding on the development of postmenopausal osteoporosis. Menopause 17: 1161-1166.

47. Wang Q, Huang Q, Zeng Y, Liang JJ, Liu SY, et al. (2016) Parity and osteoporotic fracture risk in postmenopausal women: a dose-response meta-analysis of prospective studies. Osteoporos Int 27: 319-330.

48. Carneiro RM, Prebehalla L, Tedesco MB, Sereika SM, Hugo M, et al. (2010) Lactation and bone turnover: A conundrum of marked bone loss in the setting of coupled bone turnover. J Clin Endocrinol Metab 95: $1767-1776$.

49. VanHouten JN, Dann P, Stewart AF, Watson CJ, Pollak M, et al. (2003) Mammary-specific deletion of parathyroid hormone-related protein preserves bone mass during lactation. J Clin Invest 112: 1429-1436.

50. VanHouten JN, Wysolmerski JJ (2003) Low estrogen and high parathyroid hormone-related peptide levels contribute to accelerated bone resorption and bone loss in lactating mice. Endocrinology 144: 5521-5529.

51. Chapman DJ (2012) Longer cumulative breastfeeding duration associated with improved bone strength. J Hum Lact 28: 18-19.
52. Streeten EA, Ryan KA, McBride DJ, Pollin TI, Shuldiner AR, et al. (2005) The relationship between parity and bone mineral density in women characterized by a homogeneous lifestyle and high parity. J Clin Endocrinol Metab 90: 4536-4541.

53. Wiklund PK, Xu L, Wang Q, Mikkola T, Lyytikäinen A, et al. (2012) Lactation is associated with greater maternal bone size and bone strength later in life. Osteoporosis Int 23: 1939-1945.

54. Schnatz PF, Marakovits KA, O'Sullivan DM (2010) Assessment of postmenopausal women and significant risk factors for osteoporosis. Obstet Gynecol Surv 65: 591-596.

55. Seeman E (2002) Pathogenesis of bone fragility in women and men. Lancet 359: 1841-1850.

56. Henderson PH, Sowers M, Kutzko KE, Jannausch ML (2000) Bone min eral density in grand multiparous women with extended lactation. Am J Obstet Gynecol 182: 1371-1377. 